\title{
¿Cómo se desarrolló la industria vitivinícola en Chile en la última década del siglo XX?
}

\author{
Julián Andrés Prieto Forero \\ Escuela de Negocios, Gestión y Sostenibilidad \\ Programa de Especialización en Gestión Empresarial \\ Especialista en Gestión Empresarial \\ julianprieto321@yahoo.com \\ Luis Eduardo Ibáñez Rodríguez \\ Escuela de Negocios, Gestión y Sostenibilidad \\ Programa de Especialización en Gestión Empresarial \\ Especialista en Gestión Empresarial \\ luis.ibanez.rodriguez@gmail.com
}

\section{Resumen}

El presente artículo reúne los elementos de estudio necesarios para conocer la industria del vino en Chile, su historia, comportamiento y oportunidades, que permitan explicar el crecimiento presentado en década de los años noventa.

Se quiere hacer un análisis del periodo propuesto por el crecimiento mostrado en la exportación de la industria vinícola de Chile, presentando los principales indicadores que narren la historia, reflejen la situación que vivió en ese momento y planteen las bases que dieron inicio a la industria como está en la actualidad. Se tienen en cuenta aspectos como el origen, la presencia de la industria en el territorio desde las hectáreas de cultivo hasta su participación en el producto interno bruto, la generación de empleo, participación en las exportaciones, fomento del turismo y las perspectivas que plantea la industria para su futuro cercano.

Palabras clave: Enólogo; Vitivinícola; Cepa; Naturaleza; Origen; Reserva, Vides, Commodities 


\section{Introducción}

Este articulo académico tiene carácter de informe y pretende analizar la industria del vino en el siglo XX cuando se presentó una reforma agroindustrial en el sector, "presentando al mundo en cada botella complejidad, elegancia, facilidad de consumo y un inocultable sello de innovación, todo contribuye a deleitar a quienes buscan nuevos descubrimientos"i, la década de los años noventa es un escenario muy interesante de analizar debido a los grandes cambios económicos que enfrento el mundo, entre ellos la globalización que para muchos expertos comenzó en 1989 con la disolución de la URSS, unión de repúblicas socialistas soviéticas, que impulsó el dominio del sistema capitalista y el auge del comercio internacional, este escenario mostraba nuevas formas de relación entre las naciones por lo que un evento económico podía afectar otras partes del mundo sin importar cercanía o lejanía geográfica, consecuentemente en América Latina la década de los años noventa tiene de manera homogénea la práctica de las teorías neoliberales; Guy Sorman, economista liberal francés, pregonó en los países de Latinoamérica las privatizaciones como "utopía de cambio que se impuso en estos cuatro años en todo el mundo como una necesidad indiscutible"ii. El fenómeno de la transnacionalización de las empresas adquiere importancia en los mercados y evidencia la aparición de un nuevo orden económico mundial, que supera las fronteras nacionales impidiendo que los gobiernos pudieran intervenir de manera unilateral fijando precios, cuotas de producción o regulaciones. En chile específicamente se presenta un fenómeno especial y es el regreso a la democracia que abre las fronteras que por la dictadura estaban cerradas, esto dinamiza los mercados y obliga al país a entrar en un entorno que demanda primero, especialización en la industria, segundo, creación de valor en el producto, tercero, involucramiento en la competencia de precios, y cuarto la necesidad de agremiación de sus mercados.

Por otro lado, la industria vitivinícola no es ajena a estos cambios y enfrenta los retos con la profesionalización necesaria para convertirse en un motor de desarrollo de la economía; Para iniciar su observación es necesario que fijemos unos precedentes que chile como país tiene y que enriquecen la posibilidad de la industria.

Chile es un país que cuenta con ventajas naturales para la producción del vino, tiene las cuatro estaciones, aunque sus veranos son largos y calurosos, es en donde el riego que se hace a la uva nace del deshielo de sus nevados. "Prácticamente no se cuenta con pestes y enfermedades, sus suelos son variados y hacen que se pueda cultivar la uva de vino en 14 de sus regiones a lo largo de todo su territorio"iii.

El día 4 de septiembre celebra el día nacional del vino, la industria hace parte de la oferta turística del país lo que la ha convertido luego de Francia como el destino turístico más 
importante del mundo. Se destaca su importancia convirtiendo el sector en uno de los más alabados alcanzando lugares de excelencia a nivel mundial. Según PROCHILE el 70\% del vino que se exporta proviene de viñas certificadas, $86 \%$ de las viñas posee áreas de conservación de ecosistemas y más de 6.000 de los empleados de los cultivos tienen certificación en competencias laborales.

\section{Importancia de la Industria Vinícola en Chile}

Para determinar la importancia de la industria vinícola en la economía Chilena es importante tener unos antecedentes de su evolución, los factores económicos y políticos relevantes que dieron apertura e importancia a la industria, según el portal memoria chilena de la Biblioteca Nacional de Chile ${ }^{\text {iv }}$ el vino ha sido parte de la historia económica y patrimonio del país, llegando desde su inicio a través de los conquistadores españoles en el siglo XVI, tenemos como referencia diversos personajes de la historia, Rodrigo de Araya, Francisco de Aguirre, Pedro de Cisternas, Conquistadores españoles y encomenderos quienes cultivaron los primeros viñedos y generaron vinos para consumo personal.

Transcurrieron los años y esta cultura de viñedos se extendió a lo largo del país, tanto se desarrolló la industria del vino que logro realizar sus primeras exportaciones a los países vecinos y logro posicionarse a nivel mundial como un gran exportador de vino, su éxito fue tal que la corona española protegió la compra y venta entre países de vinos españoles y decretó políticas proteccionistas para la importación de vinos provenientes de Suramérica.

En el siglo XIX, Chile logra desligarse de la corona y leyes Españolas lo cual abre la posibilidad a las familias más privilegiadas de realizar viajes a Europa para apreciar los vinos franceses dando como inicio a la importación de nuevas cepas, costumbres y tendencia que se manejaban en el viejo mundo en base a la industria y con el único fin de fomentar e implementar estos nuevos conocimientos en los valles de Maipo, Rapel y Aconcagua para producción de una mejor clase de vino comenzado así, la era moderna de producción de este sureño país.

Las inversiones realizadas por los empresarios vinicultores no solo se reflejaron en adquisición de grandes extensiones de tierra, construcción de grandes bodegas, importación de maquinaria especializada, renovación de las vasijas, innovación en cepas exportadas desde Europa especialmente de Francia y especialización de mano de obra calificada. Esto implicaba una transformación importante en la cultivación de las uvas hasta la fabricación del vino mejorando las prácticas de los nativos que trabajaban la en el cultivo de uvas, en 1860 el empleo de maquinaria especializada en la agricultura vinícola era prácticamente 
desconocida, en cambio 1863 y 1889 inicia el gran cambio realizando importaciones de más de 421 máquinas vendimiadoras especialmente de países como Estados Unidos y Francia.

Antes del siglo XX y para la época el vino no tenía tanto auge en consumo en Chile, por consiguiente tampoco era considerado como un producto de exportación, los pocos chilenos que consumían esta bebida preferían realizar importación de vinos especialmente de Europa para las familias más adineradas y sobre todo para ocasiones especiales; en cuanto a la producción de vino de bajo costo debían competir con el vino tradicional extraído de la cepa "país" que popularmente era la más usada en todo Chile.

Previo a la industrialización de los viñedos estos no generaban rentabilidad, al pasar el tiempo y a medida que el vino ganaba posicionamiento con salida al mercado de nuevos vinos, marcas y sobre todo mejorando sus canales de distribución factor clave para que el sector presentara un aumento de la en los índices de fabricación de vino entre los años 80's y 90's, ya que pasó de 41,7 millones de litros en 1883 a 243 millones en 1923.

En consecuencia que crecía la producción y el consumo de vino se vio la necesidad de mejorar el bodegaje y la distribución tanto en los centros urbanos como en zonas rurales, aquí juega un papel importante la comercialización de los productos los cuales fueron asumidos por las viñas productoras quienes en sus activos poseían flotas de autos y camiones, lo interesante es que la mayoría de esta distribución fue asumida por empresas especializadas más que todo de origen Español especialmente de la provincia de Cataluña, esto cobra sentido cuando analizamos que los Catalanes poseían más experiencia que los Chilenos en temas de distribución e identificaron una oportunidad que los productores Chilenos no explotaban de manera adecuada, algunos tomaron la iniciativa y posteriormente fueron involucrando a más empresarios catalanes a esta actividad, de esta manera formaron un cadena de producción involucrando el cultivo, la fabricación y distribución completa, uniendo etnias, conocimientos especializados por sectores y sobre todo sin entrar en conflictos con los grandes poseedores de las viñas, distribuidores, bodegueros y consumidores.

Sin lugar a duda y a lo largo de la historia este proceso contribuyo al crecimiento de los viñedos y más tarde la aparición de otros actores especializados como los bodegueros que realizaron su aporte a este proceso de renovación de la industria vinífera, pasaron de una actividad rutinaria y familiar a una actividad verdaderamente agroindustrial que movilizaba grandes capitales y contribuyó así de manera importante al desarrollo de una de los países emergentes de Suramérica, adicional con el crecimiento de la industria de vino otros sectores se vieron favorecidos de manera directa como los son la fabricación de botellas, la tonelería y sector corchero contribuyendo así al crecimiento del país en diferentes sectores. 
Con los progresos de la industria agrícola también tuvieron progresos en el capital humano y social aunque los grandes poseedores de viñedos se enfocaron en la inversión de nuevas tecnologías y conocimientos esto no estaba en la misma línea con los trabajadores en el campo, el proceso de registro de obras o faenas agrícolas seguían siendo de manera artesanal ejecutadas mayoritariamente por los trabajadores llamados como inquilinos, quienes no eran considerados como mano de obra pues sus contraprestaciones eran en alimentos, pequeñas participaciones económicas y en algunas ocasiones se beneficiaban con pequeñas viviendas en los viñedos, también estaban los trateros, estos recibían una contraprestación fija mensual en dinero con el cual subcontrataban a más personas para trabajar al interior del viñedo, generalmente estos acudían a sus familiares, el trabajo de menores era bien visto para la época no tenían consecuencias legales sobre todo en algunas temporadas del año cuando la producción presentaba incrementos.

Antes del siglo XX los trabajadores no pudieron organizar sindicatos ya que los grandes propietarios de los viñedos tenían total apoyo del gobierno dejando de lado la aplicación de leyes de protección a los trabajadores de la agricultura, de esta manera los diferentes actores en mano de obra vivían en una confusión entre paternidad y opresión por parte de sus patronos, en las zonas urbanas el manejo de personal era más parecido a mano de obra calificada pues los trabajadores debían realizar todo el procesos desde la faena hasta la distribución pasando por el embotellamiento del vino, reciben una contraprestación salarial lo que significaba su único sustento no se desligaron de la falta de garantías y leyes de protección laboral al igual que los trabajadores de la zonas rurales no podían conformar sindicatos, después de la segunda guerra mundial las huelgas de los trabajadores tomaron fuerza y es en este momento donde también surge una transformación de la dignificación laboral en los trabajadores de los viñedos permitiendo a las grandes empresas mejorar su prácticas de administración consolidándose como verdaderas empresas y dejando de ser negocios familiares el ejemplo más claro en la Viña Concha y Toro el mayor referente de Chile, puesto que aún mantiene.

A partir de los años 90’s los grandes productores y los empresarios del vino en su gran mayoría de Estados Unidos, España, Francia y Japón decidieron dar un vuelco en la industria realizando grandes inversiones en maquinaria, tecnología, materia prima, instalaciones y mejorando significativamente sus sistemas de transporte, tardaron 30 años en concluir dicha transformación y mostrando así la nueva era del de la industria vinícola chilena, consolidándose y posicionándose como uno de los principales productos en la economía del país.

A raíz de los cambios políticos dados en 1973 con la muerte de Salvador Allende y el posicionamiento en el poder por parte de Augusto Pinochet, quien dio fin al proceso de 
nacionalización y devolvió los grandes viñedos a las familias históricas ya que la producción de vino decayó por la guerra civil dada por la época pues los viñedos estaban siendo destruidos

En este artículo analizaremos esta última década del siglo XX donde la industria vinícola se convirtió en un factor clave en la economía chilena. En Latinoamérica Chile se ha convertido en las últimas décadas como un referente a seguir en cuanto a su crecimiento económico esto dado a varios factores como su apertura económica que sólo dependía de materias primas, o productos básicos que fácilmente podían ser proveídos por sus vecinos suramericanos, en Chile se ha incentivado el emprendimiento a través del aprovechamiento de sus ventajas naturales sumándole inversión en tecnología el mejor ejemplo lo encontramos en la industria Vinícola la cual se convirtió en un factor relevante en el PIB del país aportándole alrededor de 150.000 millones de dólares.

En ese sentido y siendo fieles a la tradición que caracteriza la industria y enorgullece a los chilenos, el país ha logrado consolidarse como una de los más importantes exportadores del continente americano compitiendo con grandes potencias como España, Italia, Francia y Australia, tanto es su crecimiento e importancia en el país que su crecimiento anual se calcula entre el 3\% y el 4\%, a partir del año 2000 según el portal todo vinos "la industria vinícola presento una tasa de crecimiento anual, en valor, de $8,9 \%$. En términos de volúmenes la variación total en un período de quince años fue de $20 \%$ con una tasa de crecimiento anual de $8,4 \%$ ". El precio en promedio de las exportaciones, aumentaron 7,4\%, con un valor de USD 1,96 por litro en 2001 y de USD 2,10 por litro en 2015 siendo uno de sus principales clientes el mercado chino con una participación del $40 \%$ con vino embotellado, vino a granel y espumoso.

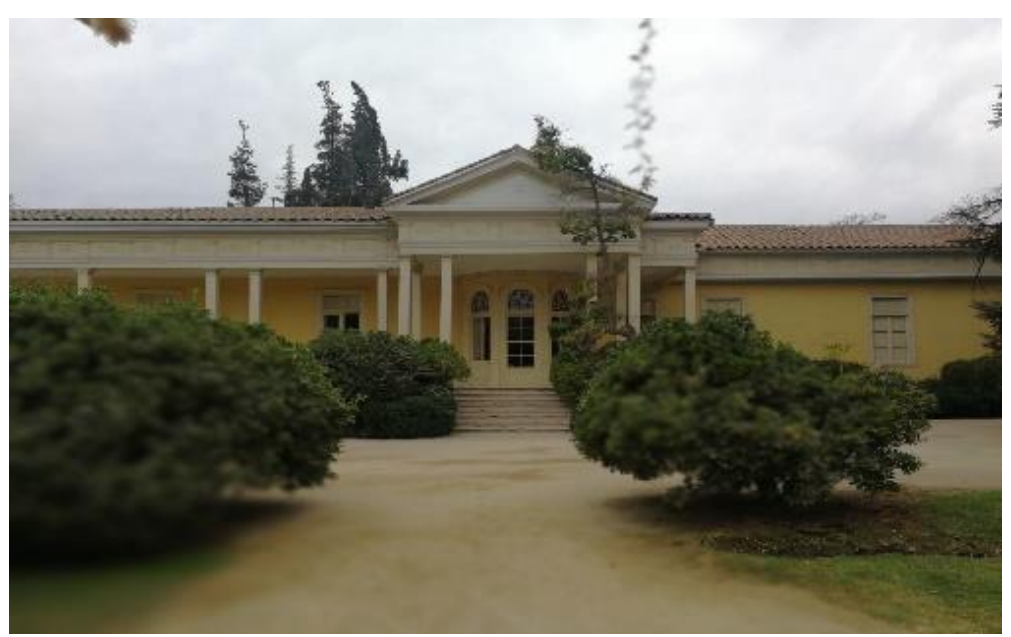

Casona Viñedo Concha y Torro Chile

Fuente: Propia (2018) 


\section{Comportamiento de la industria en cifras}

Según cifras del ministerio de agricultura de Chile ${ }^{v}$, Servicio Agrícola y Ganadero, S.A.G. ${ }^{v i}$ en la última década del siglo XX el comportamiento de las exportaciones de vino en hectolitros presento un importante crecimiento a partir del de 1995 (véase gráfico 1).

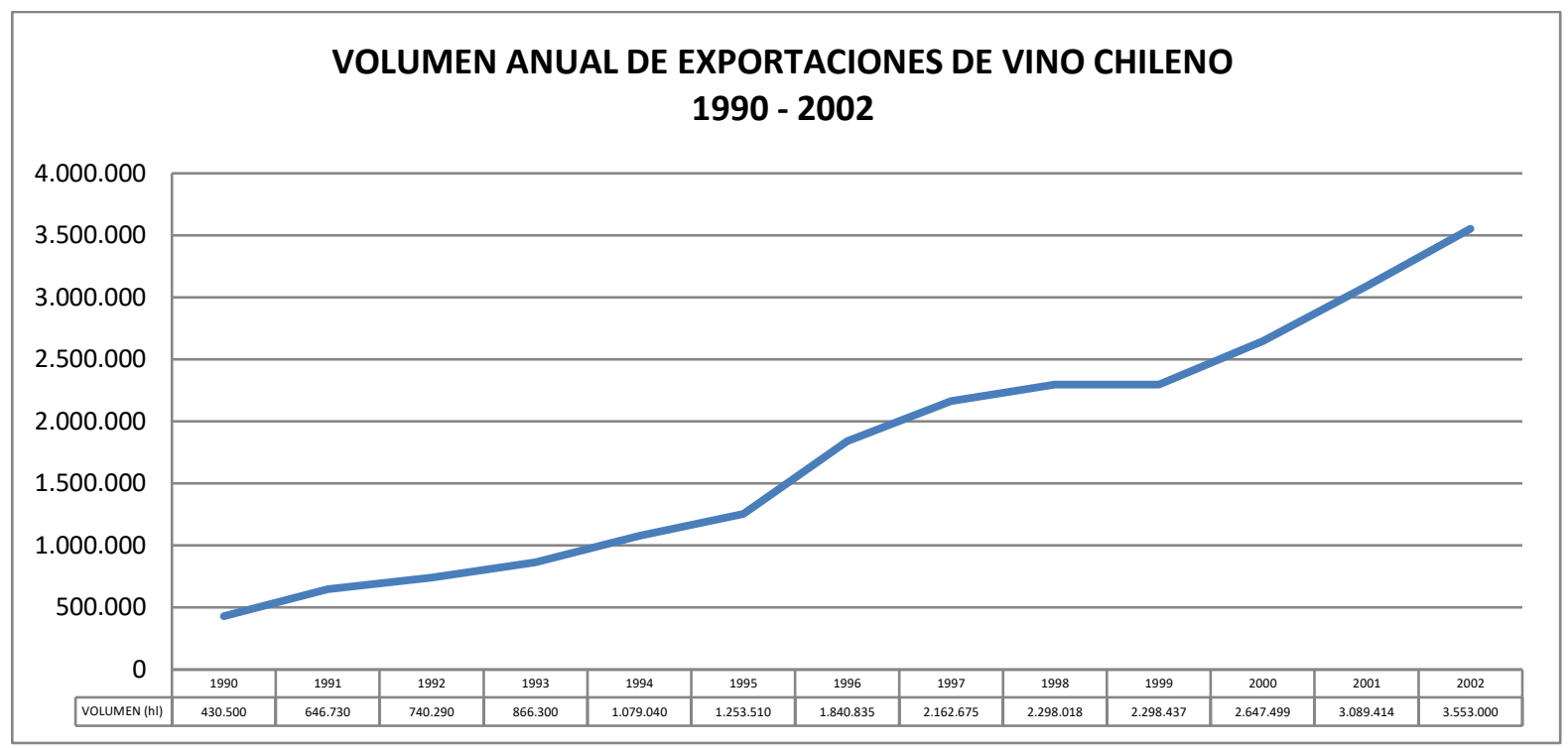

Gráfica 1 volumen anual de exportaciones

Fuente: SAG Servicio Agrícola y Ganadero - Gobierno de Chile

El aumentar en casi diez veces la exportación de vinos en una década, así como el cambio de la mezcla del destino de las exportaciones hace pensar que la política de apoyo a la industria funcionó bien para el país y se debe tener en cuenta como uno de los factores que más influyeron en el desarrollo de la industria.

De modo idéntico el artículo publicado en la biblioteca digital de la CEPAL Chile "Capacidad de innovación en industrias exportadoras de Chile ${ }^{\text {vii: }}$ la industria del vino y la agroindustria hortofrutícola" nos evidencia que la industria del vino en Chile tuvo su principal crecimiento en la época del siglo XX lo que permitió ser reconocido a nivel mundial como el quinto país con mayores ventas y posicionándose como el segundo país con mayor crecimiento en el sector, alcanzando una cota de participación de 5,3\% en el mercado mundial que representaba en ventas más de 4,5 millones de hectolitros de vino, con un valor de 834 millones de dólares, en este siglo gano mercado Europeo, Norte América y Asia, utilizando como estrategia la relación costo-calidad ofreciendo vinos de buena calidad a bajos pero esta estrategia produjo que los precios no fueran estables con las exportaciones. 
Sin lugar a duda la innovación en las empresas también fue un elemento clave en el desarrollo del sector, el factor principal fue no ver la industria como commoditie ${ }^{\text {viii }}$, es decir trasladarse de vinos llamados corrientes hacia los llamados de mayor calidad, incrementando los precios, factor clave pues se dedicaron a mejorar notablemente sus procesos enfocado en satisfacer a los consumidores ofreciéndoles diversidad de sus productos.

La mezcla que se tenía a comienzos de la década en estudio muestra que el $88 \%$ de las exportaciones se hacía a países de Latinoamérica mientras el 2\% a países de Europa, para el año 2.000 el mercado latinoamericano recibía el 11\% y Europa el 31\%, así como también se muestra un incremento importante de las exportaciones a Estados Unidos que aumentaron su participación del $10 \%$ al $33 \%$ en la misma década.

Tabla 1:

\begin{tabular}{|c|c|c|c|c|c|c|c|}
\hline \multicolumn{8}{|c|}{$\begin{array}{l}\text { EXPORTACIONES DE VINO CHILENO I } \\
1995, \\
2000,2001,2002 \text { y } 2003 \text { (\% en Volumen) }\end{array}$} \\
\hline MERCADO & 1980 & 1985 & 1990 & 1995 & 2000 & 2001 & 2002 \\
\hline LATINOAMERICA & $88 \%$ & $70 \%$ & $37 \%$ & $23 \%$ & $11 \%$ & $9,70 \%$ & $7,80 \%$ \\
\hline \multicolumn{8}{|l|}{ Y МЕХICO } \\
\hline EUROPA & $2 \%$ & $3 \%$ & $17 \%$ & $31 \%$ & $48 \%$ & $52,20 \%$ & $52,80 \%$ \\
\hline ASIA-OCEANIA & 0 & 0 & 0 & $3 \%$ & $6 \%$ & $9,20 \%$ & $12,30 \%$ \\
\hline \multirow[t]{2}{*}{ OTROS } & $0 \%$ & $0 \%$ & $2 \%$ & $4 \%$ & $1 \%$ & $0,30 \%$ & $0,20 \%$ \\
\hline & $100 \%$ & $100 \%$ & $100 \%$ & $100 \%$ & $100 \%$ & $100 \%$ & $100 \%$ \\
\hline
\end{tabular}

Tabla 1 Distribución porcentual

Fuente: SAG Servicio Agrícola y Ganadero - Gobierno de Chile

Al interior es importante tener en cuenta la evolución de la superficie sembrada en tres principales productos de la industria, una primera que es la una de la que se extraen los vinos o de vinificación, una segunda clase que son las uvas comestibles en mesa y por último una tercera clase que es la uva con la que se fabrican los piscos o uva pisquera entre 1985 y 2003 en hectáreas ${ }^{\mathrm{ix}}$, estas cifras igualmente las suministra el ministerio de agricultura y son fuente de nuestro análisis. 
En el siguiente grafico (véase gráfico 3) nos muestra la evolución de las hectáreas cultivadas en estos tres diferentes productos, el primero es la uva de vinificación, que es la que sirve de fuente de nuestro análisis, pero la comparación con la uva de mesa y la uva pisquera es muy valiosa para verificar el interés de los cultivadores por participar de una industria creciente que aumenta de manera más fuerte que las otras.

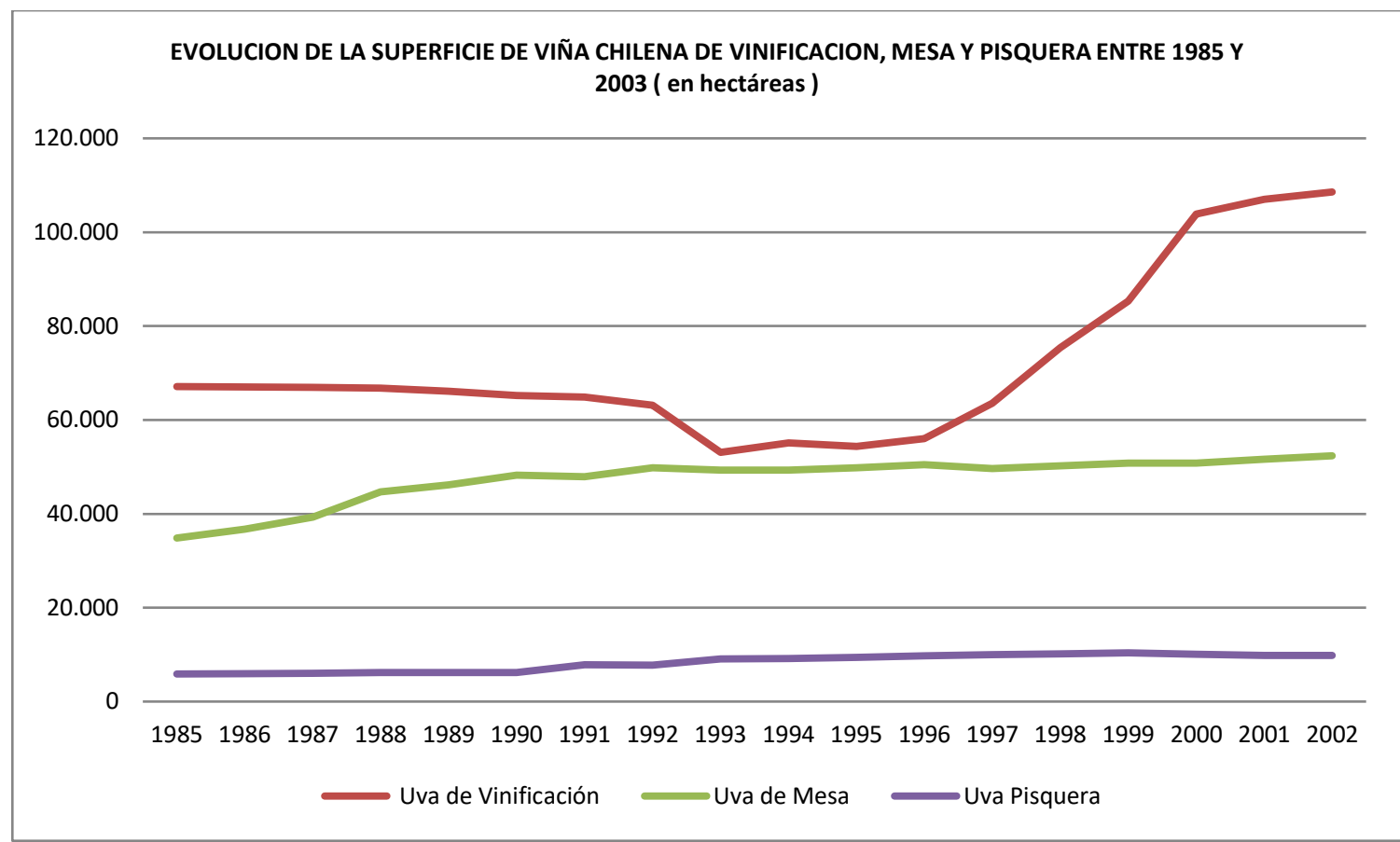

Gráfica 2 Evolución

Fuente: SAG Servicio Agrícola y Ganadero - Gobierno de Chile.

De acuerdo con la afirmación de la unión europea en su estudio "El Sector Vitivinicola en Chile" "la superficie de viñedos se ha incrementado sostenidamente hasta llegar a más que duplicarse entre 1995 y 2005. Igualmente se anota que se ha venido concentrando la producción en vinos con denominación de origen, a estos se les llama así porque representan por su calidad o características una región en particular de un país, en 1996 estos representaban el 55.7\% del volumen de vinos producido en el país, en 2.006 llegaron a representar el $85 \%$ de la producción chilena de vinos.

Chile destina el $0.68 \%$ de su producto interno bruto a investigación y desarrollo, Brasil ocupa el primer lugar en Latinoamérica en este aspecto, según cifras del mismo estudio, el sector público respalda el 53\% de la inversión en investigación y desarrollo, el sector privado provee el $37 \%$ y un $10 \%$ de otras fuentes. 
Los recursos recibidos se utilizan en un $46 \%$ en actividades netamente de investigación y desarrollo que generan las empresas, las universidades proponen el $32 \%$ y un $10 \%$ por organismos públicos y en un $12 \%$ por instituciones privadas sin ánimo de lucro; La investigación básica es el $22 \%$ y la aplicada y desarrollo un $78 \%$ del total.

Chile cuenta con 8.507 investigadores en total, de los que 6.476 ejercen en universidades, 964 en las empresas del sector, 506 en organismos públicos, 413 en instituciones privadas sin ánimo de lucro y 148 en otros sectores. eso garantiza recursos importantes en el sector vitivinícola que reúnen diferentes entidades alrededor del desarrollo de la industria; La Ingeniera Katrina Muller, de la facultad de ciencias agronómicas de la universidad de Chile y quien es enóloga ${ }^{x i}$, menciona que el apoyo de asociaciones e instituciones impulsa la industria en chile, el noventa por ciento del vino embotellado y del mercado interno está apoyado por Chile Vid, creada en 1.992 y que congrega 42 viñas y Viñas de Chile que concentra otras 45 viñas, estas dos organizaciones están representadas en el exterior (EEUU y Gran Bretaña) por Wines of Chile fundada en 2.002 .

Prochile igualmente cumple un papel fundamental y es una agencia gubernamental dependiente del ministerio de relaciones exteriores de Chile, El S.A.G. (Servicio Agrícola y Ganadero) dependiente del ministerio de agricultura chileno y quien entre otros objetivos contribuye a garantizar la calidad de los vinos que se producen garantizando cumplir con las imposiciones de los países a los que llega.

La corporación Chilena del Vino que como asociación gremial representa al sector ante instituciones gubernamentales y privadas, así como las universidades que forman enólogos y llevan a cabo investigación como lo son: Universidad Católica de Chile, Universidad de Talca y Universidad de Chile. Importante tener en cuenta que para ser enólogo se debe ser ingeniero agrónomo titulado, con especialidad en enología y viticultura y aprobar una vez al año el examen que realiza la asociación de ingenieros agrónomos de Chile.

De acuerdo al artículo del enólogo Philippo Pszczólkowski de la universidad católica de chile, xiic En Chile, se tuvo un incremento en las exportaciones de vino debido a la salida de Pinochet del poder que permitió el ingreso a mercados que mantenían un bloqueo a los productos chilenos, este incremento se ve reflejado al pasar de 30 millones de dólares en 1990 a 600 millones en 2.003 y poco más de 1.800 en 2.014 .

En los años noventa los vinos de Chile lograron posicionarse con firmeza en el mercado mundial, en consecuencia y acorde a los datos del grafico 4 (véase gráfico 4) podemos asegurar que Chile es un país con tendencia creciente en la producción y exportación de vino 
en el período estudiado, que contribuyó notoriamente al desarrollo de la investigación en el mismo campo y creo las bases de la cooperación gremial que permite un avance en los indicadores del sector ${ }^{x i i}$.

Una de las consecuencias lógicas de darse a conocer con productos diferenciales de alta calidad en todo el mundo es el flujo de visitantes a las viñas que por diferentes factores hacen del enoturismo una industria anexa que se abre a las nuevas oportunidades de negocio, de igual manera se debe entender que el visitante busca una experiencia que lo identifique con el vino, quizá su vino favorito y por tanto la industria chilena crea las llamadas rutas del vino que se extienden por los valles de Colchagua, Casablanca y Maipo principalmente, La colaboración estrecha entre las áreas de turismo de las viñas, las agencias de turismo y los operadores logró el incremento notorio de visitas y permitió vislumbrar las posibilidades de este nuevo negocio. Concha y Toro, fundada a finales del siglo XIX, se encuentra entre las rutas más frecuentadas, entre otras cosas por el reconocimiento de sus marcas, entre las cuales sobresale Casillero del Diablo, introducido en el mercado mundial hace 62 años. Exportado a 137 países, el Casillero del Diablo es la marca chilena más reconocida en el mundo.

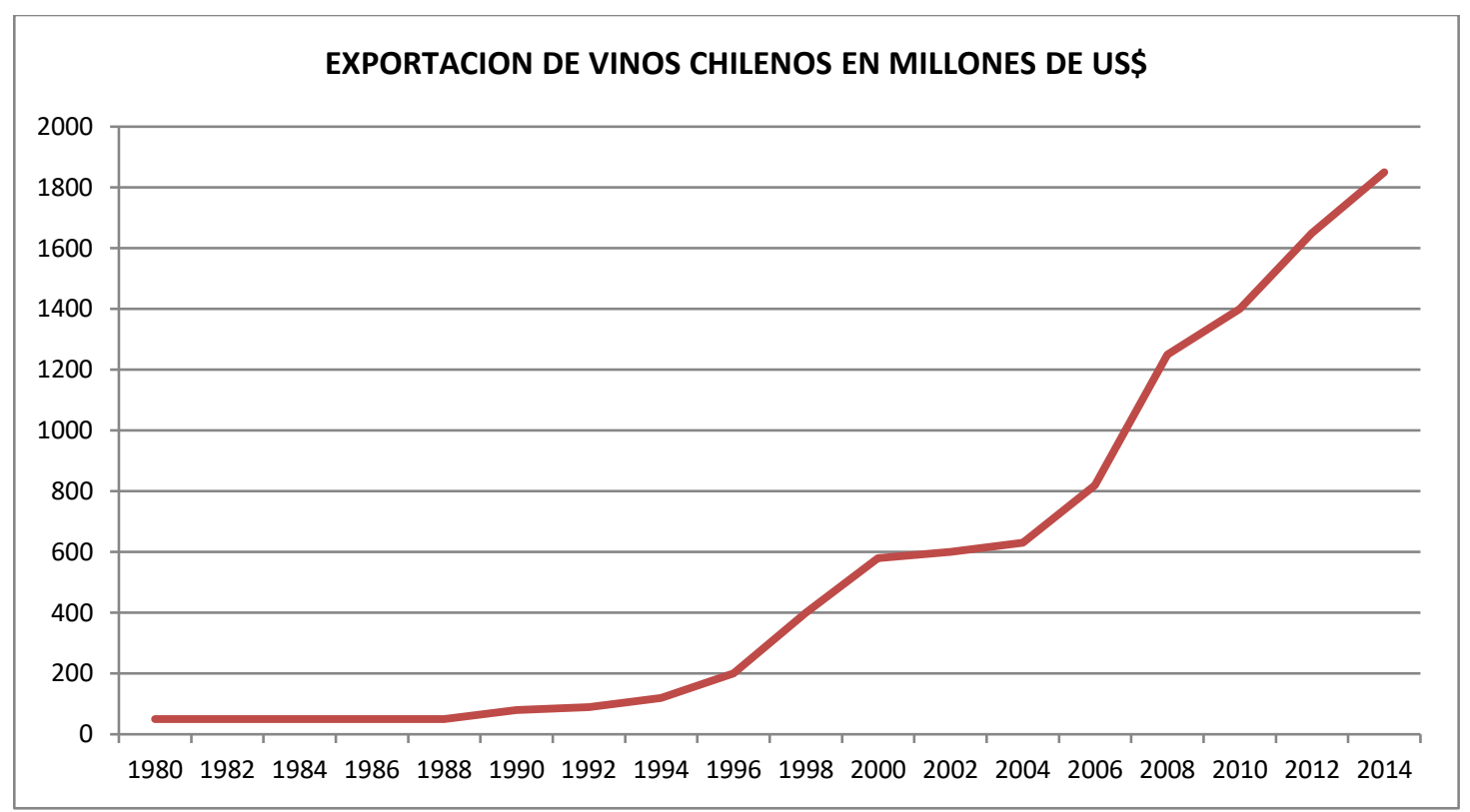

Gráfica 3 Exportación de vinos chilenos

Fuente: SAG Servicio Agrícola y Ganadero - Gobierno de Chile.

Su nombre se deriva del rumor dispersado por su propio fundador, don Melchor de Concha y Toro, para evitar que misteriosas manos sacaron sin su autorización vinos de su cava personal. Para defenderse del saqueo, don Melchor inventó la fábula de que el diablo se había 
instalado en su bodega y, por tanto, era un riesgo meterse allí. Desde entonces no volvió a perderse ni una sola gota de vino de su casillero, dando origen a una leyenda.

Las visitas realizadas a los viñedos estuvieron acompañadas de degustaciones que como en la mayoría de los países en donde el turismo de este tipo se realiza se hacen de manera gratuita, cabe anotar que en países europeos este ramo del turismo se lleva a cabo desde hace más de dos décadas pero que para el caso chileno no representaba una utilidad en sí, lo que llevó a cobrar una entrada a los turistas que les permitiera no sólo degustaciones de los vinos tradicionales o de la línea básica sino también se pudieron ofrecer vinos Premium que ampliaron las opciones de degustación. ${ }^{\text {xiv }}$

\subsection{Cifras Nacionales}

De acuerdo con datos de la CEPAL, el índice de producción agrícola en esta década se comportó de manera favorable tal como lo indica la tabla 2:

\begin{tabular}{l|l} 
Años & valor \\
\hline 1990 & 58,87 \\
1991 & 60,62 \\
1992 & 63,33 \\
1993 & 67,21 \\
1994 & 72,35 \\
1995 & 76,53 \\
1996 & 79,27 \\
1997 & 79,3 \\
1998 & 81,47 \\
1999 & 80,16 \\
2000 & 82,49
\end{tabular}

Gráfica 4 Índice de producción agrícola

Fuente: Cepal

En la agricultura, el índice de producción agrícola o de la producción por unidad de tierra, es el comúnmente más utilizado para dar una idea de la eficiencia o rendimiento de los cultivos. El desarrollo del rendimiento a lo largo del tiempo como lo muestra el indicador generado para Chile en la década de estudio se utiliza en muchos casos como indicador de una mayor eficiencia económica, en Chile la agricultura sostenible es aquella que gestiona y usa el ecosistema agrario de manera que preserva la diversidad biológica, el rendimiento de los suelos no puede ser sinónimo de deforestación y uso de pesticidas; La productividad, la capacidad de regeneración y la vitalidad tanto en el presente como en el futuro sin dañar otros 
ecosistemas son objetivos claros de los gremios agrícolas y su tecnificación y profesionalización son tarea de los agricultores que a diario enfrentan nuevos retos, entre ellos en la actualidad el cambio climático.

De igual forma se ve reflejado el beneficio en el índice de desempleo a nivel nacional que presenta indicadores por debajo de los dos dígitos y estables, como se presenta en la siguiente tabla:

\begin{tabular}{l|l} 
Años & valor \\
\hline 1990 & 7,80 \\
1991 & 8,20 \\
1992 & 6,70 \\
1993 & 6,50 \\
1994 & 7,80 \\
1995 & 7,40 \\
1996 & 6,40 \\
1997 & 6,10 \\
1998 & 6,40 \\
1999 & 10,10 \\
2000 & 9,67
\end{tabular}

Gráfica 5 Índice de desempleo

Fuente: Cepal

Es difícil asegurar que un sector en particular pueda tener incidencia directa en el indicador nacional de tasas de desocupación, de acuerdo a SOFOFA la industria vitivinícola en Chile que es una agremiación que agrupa a diferentes empresas del sector industrial chileno ${ }^{\mathrm{xv}}$, la industria del vino emplea a más de 110 mil personas de forma directa, representando el 15\% de la oferta de empleo en el agro, pero el dato nos parece relevante teniendo en cuenta que la economía chilena es en gran parte dependiente de la extracción del cobre y que esa actividad no es lo suficientemente generadora de empleos como para creer que el comportamiento del PIB se pueda ver reflejado directamente en la ocupación como dato nacional.

Según Datos de la CEPAL. El desarrollo económico en América Latina tuvo un cambio de directriz al comenzar la década de los años noventa, uno de los indicadores más afectado fue el de desempleo que tuvo un incremento en sus índices llegando alrededor del 10\%. . "en "en países como Colombia, Uruguay y Argentina se presentaron índices del 16,5\%, 17\% y 19,7\% respectivamente, en general todos los países sudamericanos tuvieron incrementos y por otro lado el empleo formal disminuyó como porcentaje de la fuerza de trabajo en todos los países". 
En consecuencia, los datos que se presentan de la evolución del desempleo en la década para Chile presentan los mismos comportamientos de sus vecinos latinoamericanos con una fuerte tendencia al crecimiento al final de la década.

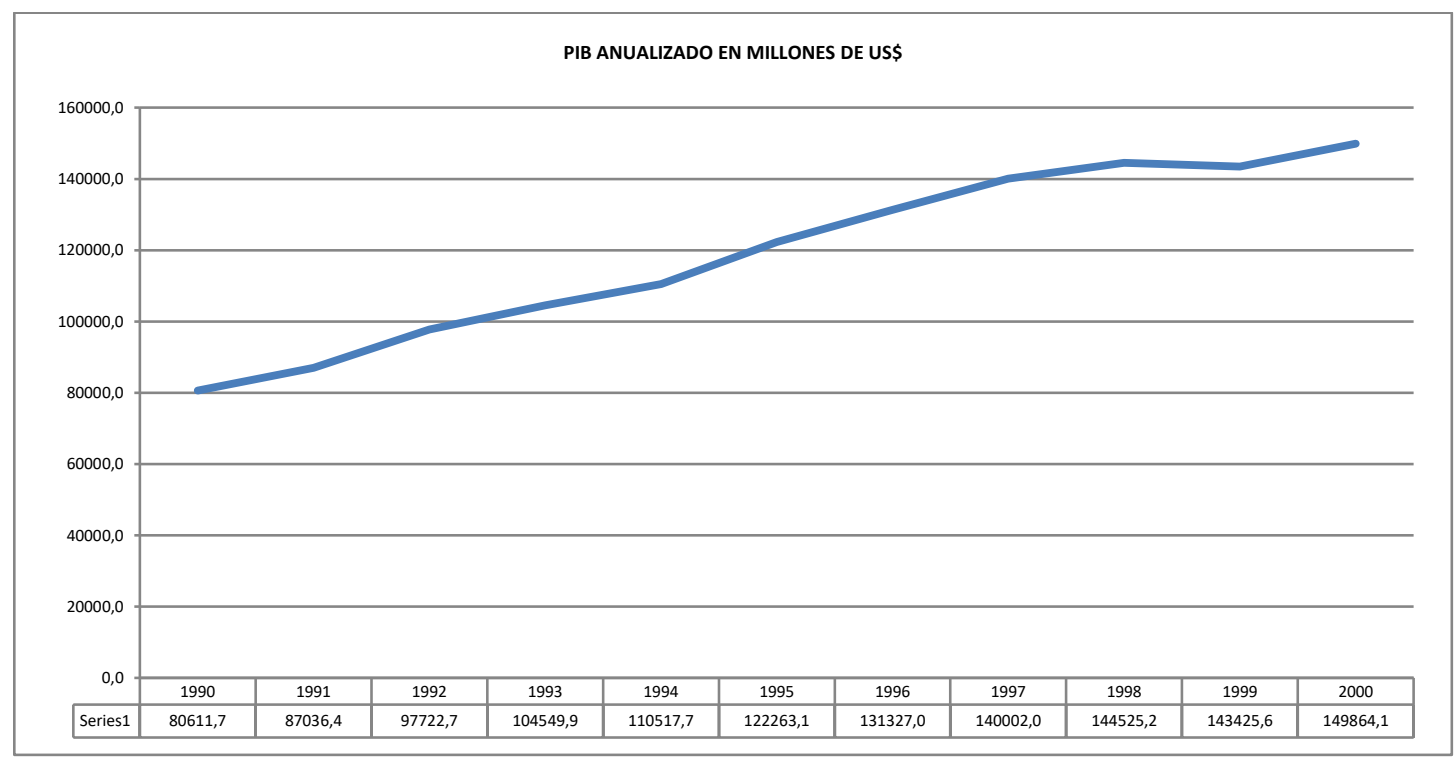

Gráfica 6 Evolución del desempleo

Fuente: Cepal

Mientras el PIB nacional de Chile termina la década en \$149.864 millones de dólares, Colombia lo hace con \$192.491 millones de dólares, dato que contrasta con la cantidad de población de ambos países.

\subsection{Comparativo de industrias vitivinícolas a nivel mundial}

La década de estudio presentó un cambio en el protagonismo de los países exportadores; A los productores y exportadores del viejo mundo como los son Italia, España, Portugal y Francia en primera línea se adicionaron los llamados del nuevo mundo, entre los que se encuentran Australia, Nueva Zelanda, EEUU - básicamente California - Argentina, Chile, Uruguay y Sudáfrica, que contaban con grandes firmas empresariales y una estructura organizativa muy competitiva.

Los viñedos europeos tradicionales han perdido terreno frente a la nueva oferta de países emergentes en la industria, los impulsos recibidos en las nuevas zonas han desarrollado un cambio significativo en la elaboración, uno de ellos fue la constitución de denominaciones de origen que permiten distinguir entre unos vinos y otros, las industrias emergentes del continente americano más importantes son Argentina, Chile y la costa este de Estados Unidos. 
En el siguiente cuadro se muestran los principales países productores de vino a nivel mundial en la década de estudio, como se puede observar Chile ingresa al grupo de los que tiene el $13 \%$ de la producción mundial junto con Alemania, África del Sur, Portugal y Rumanía.

\section{Tabla 2 Países productores de vino}

\begin{tabular}{|c|c|c|c|}
\hline \multicolumn{4}{|c|}{$\begin{array}{l}\text { PAISES PRODUCTORES DE } \\
\text { DECADA DE LOS AÑOS } 90\end{array}$} \\
\hline Más de & De 10 a & De 5 a 10 & De 2 a 5 \\
\hline $35 \quad M$ & 35 M Hl. & M Hl. & M Hl. \\
\hline \\
\hline $50 \%$ & $21 \%$ & $13 \%$ & $9 \%$ \\
\hline \multirow{8}{*}{$\begin{array}{l}\text { España } \\
\text { Francia } \\
\text { Italia }\end{array}$} & EEUU & Alemania & Rusia \\
\hline & Argentina & & Hungría \\
\hline & Australia & $\begin{array}{l}\text { Africa } \\
\text { del Sur }\end{array}$ & Brasil \\
\hline & China & Portugal & Grecia \\
\hline & & Chile & Austria \\
\hline & & & Ucrania \\
\hline & & Rumanía & Moldavia \\
\hline & & & Croacia \\
\hline
\end{tabular}

Fuente: Fundación Dinastía Vivanco.

La fundación dinastía Vivanco ${ }^{\text {xvii }}$ encarga este estudio para mostrar el mundo del vino y entre las características que mencionan se encuentran las certificaciones que se hacen de las diferentes denominaciones de origen y la sana metodología de producción al no ser atacado por plagas.

Es en la década de los años noventa que la industria muestra una actividad propia de una industria creciente, logrando que a mediados de la década la representatividad de los vinos del viejo mundo pierda participación en el mercado mundial, pasando del 72\% entre 1991 y 1995 a $57.4 \%$ en 2.010. Por su parte los países del nuevo mundo pasaron del $9.3 \%$ al $29.4 \%$ en el mismo período.

De igual forma los precios ayudaron mucho a este posicionamiento ya que los países del nuevo mundo vienen manteniendo precios competitivos sin que medie el aumento considerable de sus ventas, aumentando así su cuota de mercado de países como Australia, Chile, EEUU y Sudáfrica principalmente en Europa, acompañando estas ventajas con estrategias de marketing que afianzan la confianza del consumidor. 


\subsection{Retos}

De acuerdo con SOFOFA como agrupación gremial, la industria del vino llega a más de 150 países, genera ingresos por más de 1.470 millones de dólares al año, tiene el $2.1 \%$ de las exportaciones totales de chile, se convirtió en el sexto país mayor productor de vino y cuarto exportador; Datos que a simple vista hacen pensar que las estrategias que se utilicen para las próximas décadas deben estar encaminadas al crecimiento y fortalecimiento de la industria.

La industria se ve enfrentada a diferentes retos entre los que encuentra el desarrollo sustentable como una de las formas de producción que con mayor responsabilidad se trabajan que busque competir y diferenciarse ${ }^{\mathrm{xviii}}$. Para SOFOFA y con el fin de dar respuesta a las exigencias que el mercado internacional requiere, se deben adoptar medidas ambientales, económicas y sociales y anticiparse a los competidores ${ }^{\mathrm{xix}}$.

Esto se logra con la implementación de proyectos de investigación, desarrollo e innovación que trabajen temas de mejoramiento de la producción en calidad, mecanismos para enfrentar el cambio climático, gestión de aguas, pesticidas y energía, compartiendo buenas prácticas en los cultivos, monitoreando instrumentos internacionales, implementando responsabilidad social en la organización.

El objetivo final de estas estrategias es identificar posibles riesgos ambientales, sociales, económicos y reputacionales, que con debido tratamiento lleven la industria no solo a mantener los mercados actuales sino ingresar a nuevos, certificar el $100 \%$ de las viñas con el uso de buenas prácticas y cumplir con el compromiso del año 2.020 de ser el país productos número uno del nuevo mundo, fortaleciendo la imagen del vino chileno en el mundo y su precio promedio.

\section{Conclusiones}

\subsection{Importancia de la Industria Vinícola en Chile}

Es de destacar que la industria del vino en Chile cobra gran importancia no solamente en lo económico si no en lo social y cultural, su transformación y evolución a través de la historia ayudo a consolidar la industria en lo que es en la actualidad posicionando a Chile como el cuarto país que mayormente exporta vino, también es de resaltar como pasaron de una actividad netamente de familias adineradas que producían vino para consumo personal a un proceso de agroindustria realizando inversión en materia prima, instalaciones, mejorando sus procesos de distribución y bodegaje, realizando mejoras en mano de obra calificada y sobre todo superando los conflictos políticos, como país supieron posicionarse como un referente a nivel continental presentando tal vez los mejores índices de crecimiento económico, 
lograron entender que para superar la barreras que presentaba la industria debían realizar alianzas estratégicas con diferentes actores que poseían mayores conocimiento es el caso de mejoraron la calidad de sus vinos gracias a las influencias francesas, mejoraron su proceso de distribución y bodegaje gracias a los españoles en especial a los empresarios de la región de Cataluña, también es de resaltar como uniendo todos estos conocimiento y esfuerzos Chile como país logra sobresalir y competir con países que se pensarían son más calificados en el sector como Francia, Australia Estados Unidos entre otros, ayudando a la independencia de la economía basada en la explotación de cobre, contribuyendo al enriquecimiento de los índices económicos del país y generando desarrollo no solamente en base al vino si no en todas las industrias vinculadas como la industria de los corchos y productores de botellas.

\subsection{Comportamiento de la industria en cifras}

Teniendo como referente los indicadores del ministerio de agricultura de Chile, Servicio Agrícola y Ganadero, S.A.G. en la última década del siglo XX Chile tuvo un crecimiento exponencial en sus exportaciones aumentándola en casi diez veces, esto gracias a la unión de los sectores privados y públicos que lograron formular estrategias efectivas para dar crecimiento a la industria

posicionando en el siglo XX como el quinto país con mayores ventas a nivel mundial y el segundo con mayor crecimiento en el sector abriéndose camino en mercados competitivos como lo son el europeo y el asiático manejando una estrategia básica en relación costo calidad, gracias a esto Chile paso de exportar el $88 \%$ de sus vinos hacía a países de Latinoamérica y el $2 \%$ a países de Europeos a exportar el $11 \%$ a países latinoamericanos y el $31 \%$ a países europeos y logrando exportaciones a Estados Unidos iniciando con una participación del $10 \%$ al $33 \%$.

Un papel importante para que Chile presente cifras tan importantes de crecimiento es la innovación de las empresas la no ver la industria como commodities, es decir trasladarse de vinos de línea básica hacia los de mayor calidad o vinos de origen, subiendo los precios y satisfaciendo principalmente las necesidades de sus clientes a través de la diversificación de productos

\subsection{Crecimiento de la industria}

La década de los años noventa deja en la industria el gran crecimiento de su participación internacional, el prestigio de sus cepas en los mejores escenarios a nivel mundial, el reconocimiento por precios, calidad, tratamiento con excelencia en la producción y apertura de mercados con apoyo gubernamental y cooperación gremial ${ }^{\mathrm{xx}}$. 
Reconocer sus fortalezas en lo geográfico, amplia variedad de cepas reconocidas y descubiertas ${ }^{\mathrm{xxi}}$ casi sin presencia de plagas y enfermedades, la posibilidad de hacer presencia en ferias a nivel mundial llevando lo mejor de la producción gracias los acercamientos entre industriales y creación de gremios, poner en práctica la investigación que la academia entrega y el apoyo que los privados ofrecen; son en general las herramientas que utiliza la industria para desarrollarse plenamente en este periodo de estudio y deja en el ambiente la utilidad de poner en práctica planes estratégicos de sustentabilidad y desarrollo que involucren estamentos de toda índole en búsqueda del bienestar común ${ }^{\text {xxii }}$.

\subsection{Presencia internacional}

El que los vinos chilenos lleguen a 150 países y hayan tomado gran parte del mercado que ocupaban las tradicionales viñas europeas hace pensar que algo se hizo bien en esta década y que contribuyó al crecimiento de la industria.

La creación de Chile Vid en 1.992 y que congrega 42 viñas y Viñas de Chile que agrupa 45 viñas responsables del noventa por ciento de las exportaciones de vino embotellado, son dos de las principales herramientas que utilizan los productores para llegar a su mercado internacional $^{\mathrm{xxiii}}$, la participación de ferias a nivel mundial, la certificación de sus viñedos en las buenas prácticas de producción, el apoyo de Prochile, como parte gubernamental en la búsqueda de mayores grupos exportadores que cumplan con los estándares internacionales y la exigencia del mercado hacen que para la década el crecimiento no dependa del beneplácito de los países en su re ingreso a la democracia sino más bien de un producto fuerte que compite con los mejores y que tiene la tradición necesaria para llevar su prestigio al mundo. 


\section{Bibliografía}

\footnotetext{
i Según Sabogal Hugo, 2015, [En Línea] Nuevos enlaces chilenos [consulta, 20/31 de mayo de 2018]. Disponible en: https://www.elespectador.com/opinion/nuevos-lances-chilenos-columna-558271

ii Fernando Ariel Bonfanti, 2013 [En Línea], El escenario económico internacional en la década del '90 y durante la última crisis financiera. incidencias en la economía argentina y latinoamericana [consulta, 20/31 de mayo de 2018]. Disponible en: http://rcci.net/globalizacion/2013/fg1602.htm
}

iii Julian Dowling editor de bUSINESS Chile, 2012 "Prácticamente no se cuenta con pestes y enfermedades, sus suelos son
variados y hacen que se pueda cultivar la uva de vino en 14 de sus regiones a lo largo de todo su territorio" [consulta, 20/31
de mayo de 2018]. Disponible en: https://www.amchamchile.cl/2012/08/la-industria-vitivinicola-de-chile-calidad-
premium/

iv Portal Memoria Chilena, 2018, [en línea], La cultura del vino en chile" [consulta, 20/31 de mayo de 2018]. Disponible en: http://www.memoriachilena.cl/602/w3-article-3511.html

vi Costa Barros, Victor, 2004, [En Línea] "la vitivinicultura mundial y la situación chilena en 2004. gobierno de chile, ministerio de agricultura s.a.g. [consulta, 20/31 de mayo de 2018]. Disponible en:

https://www.sag.gob.cl/sites/default/files/LA\%20VITINICULTURA\%20\%20MUNDIAL\%20Y\%20LA\%20SITUACION \%20CHILENA\%20EN\%202004.pdf

vii Graciela Moguillansky, Juan Carlos, Salas Gabriela Cares, 2006 [En Línea]“Capacidad de innovación en industrias exportadoras de Chile, la industria del vino y la agroindustria hortofrutícola” . [consulta, 20/31 de mayo de 2018]. Disponible en: https://repositorio.cepal.org/bitstream/handle/11362/4421/S0600792_es.pdf?sequence=1\&isAllowed=y

viii Graciela Moguillansky, Juan Carlos Salas, Gabriela Cares, 2006 [En Línea]“Capacidad de innovación en industrias exportadoras de Chile: la industria del vino y la agroindustria hortofrutícola”. [consulta, 20/31 de mayo de 2018]. Disponible en: https://repositorio.cepal.org/bitstream/handle/11362/4421/S0600792 es.pdf?sequence=1\&isAllowed=y

\footnotetext{
${ }^{\text {ix }}$ Costa Barros, Victor, 2004,[En Línea] la vitivinicultura mundial y la situación chilena en 2004.. [consulta, 20/31 de mayo de 2018]. Disponible en: https://www.sag.gob.cl/sites/default/files/LA\%20VITINICULTURA\%20\%20MUNDIAL\%20Y\%20LA\%20SITUACION \%20CHILENA\%20EN\%202004.pdf
}

${ }^{x}$ FOFDEF Fondo de fomento al desarrollo científico y tecnológico, 2005, [En línea], investigación al servicio de la industria vitivinícola [consulta, 20/31 de mayo de 2018]. Disponible en: http://www.conicyt.cl/fondef/2005/04/15/investigacion-al-servicio-de-la-industria-vitivinicola/

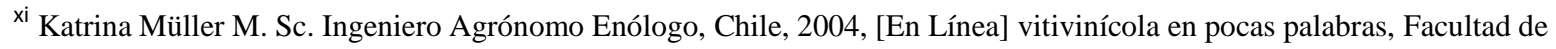
Ciencias Agronómicas. Departamento de Agroindustria y Enología, Universidad de Chile) [consulta, 20/31 de mayo de 2018]. Disponible en:

http://www.gie.uchile.cl/pdf/Katrina\%20Muller/Chile\%20Vitivin\%EDcola\%20en\%20pocas\%20palabras.pdf

xii ) Philippo Pszczólkowsk, 2016, "La Cót o Malbec en Chile y Argentina" / "The Cót or Malbec in Chile and Argentina". RIVAR Vol. 3 N ${ }^{\circ}$ 7, ISSN 0719-4994, IDEA-USACH, Santiago de Chile, enero 2016, pp. 58-80. [consulta, 20/31 de mayo de 2018]. Disponible en: http://revistarivar.cl/images/vol3-n7/4.Pszczolkowski,Phillipo.pdf 


\begin{abstract}
xiii Ministerio de Relaciones Exteriores de Chile, 2009, [En Línea], 20 años de relaciones comerciales, Dirección general de relaciones económicas internacionales, Ministerio de relaciones exteriores de Chile noviembre 2009 . [consulta, 20/31 de mayo de 2018]. https://www.direcon.gob.cl/wp-content/uploads/2013/09/Chile-20-a\%C3\%B1os-de-negociacionescomerciales.pdf
\end{abstract}

xiv Morales Sepulveda, Maximiliano;2012, [En Línea] La reingeniería del enoturismo chileno" [consulta, 20/31 de mayo de 2018]. Disponible en: https://www.americaeconomia.com/analisis-opinion/la-reingieneria-del-enoturismochileno

${ }^{x v}$ Vinos de Chile, inw of Chile, 2018, [En Línea], Codigo Nacional de Sustentabilidad, [consulta, 20/31 de mayo de 2018]. Disponible en: www.sustentavid.org

xvi Jaime Ros, 2005, [En Línea] "El desempleo en América Latina desde 1990 Unidad de Desarrollo económico s e r i e estudios y perspectivas, Cepal México, D. F., [consulta, 20/31 de mayo de 2018]. Disponible en: http://www20.iadb.org/intal/catalogo/PE/2007/01049.pdf

xvii Barco Royo, Emilio, 2003 [En Línea]“Fundación Dinastía Vivanco, El Mundo del Vino”2.003[consulta, 20/31 de mayo de 2018]. Disponible en: https://es.scribd.com/document/85956470/El-Mundo-Del-Vino

xviii This is Chile, 2014, [En Línea] La ruta del vino sustentable en Chile [consulta, 20/31 de mayo de 2018]. Disponible en: https://www.thisischile.cl/la-ruta-de-la-produccion-de-vino-sustentable-en-chile/

xix ODEPA Oficina de estudios y políticas agrarias, 2016, [En Línea], Grandes Viñas de Chile apoyarán desarrollo sustentable de pequeños productores vitícolas [consulta, 20/31 de mayo de 2018]. Disponible en:

http://www.odepa.gob.cl/publicaciones/noticias/noticias-institucionales/grandes-vinas-de-chile-apoyaran-desarrollosustentable-de-pequenos-productores-viticolas

${ }^{x x}$ Universidad de chile instituto de estudios internacionales magíster en estrategia internacional y política comercial, 2015, [En Línea], exportación del vino chileno. la estrategia de la asociación gremial vinos - chile a.g., [consulta, 20/31 de mayo de 2018]. Disponible en: http://repositorio.uchile.cl/bitstream/handle/2250/136814/Exportacion-del-vino-chileno-AlAttrach-Tabla\%20de\%20contenido.pdf?sequence $=2$

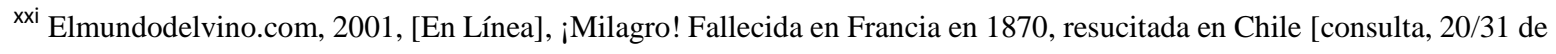
mayo de 2018]. Disponible en: http://elmundovino.elmundo.es/elmundovino/noticia.html?vi_seccion=11\&vs_fecha=200110\&vs_noticia=1003950233

xxii Coagra, 2017, [En Línea], Industria vitivinícola: Problemáticas y Desafíos, [consulta, 20/31 de mayo de 2018]. Disponible en: https://www.coagra.com/noticias/revista-coagra/articulos-destacados/industria-vitivinicola-problematicasy-desafios junio de 2017

xxiii Sanhueza, Eduardo, 2010, [En Línea], Propuesta Global de Desarrollo de la Vitivinicultura, instituto de investigaciones agropecuarias, [consulta, 20/31 de mayo de 2018]. Disponible en: http://www2.inia.cl/medios/raihuen/Comunicado_Prensa/Propuesta_Vitivinicultura.pdf 